\title{
Measurements of neutron scattering angular distributions with a new scintillator setup
}

\author{
Elisa Pirovano ${ }^{1}$, Roland Beyer ${ }^{2}$, Arnd Junghans $^{2}$, Ralf Nolte $^{3}$, Markus Nyman $^{1}$, and Arjan Plompen ${ }^{1}$ \\ ${ }^{1}$ European Commission, Joint Research Centre, Retieseweg 111, 2440 Geel, Belgium \\ 2 Helmholtz-Zentrum Dresden-Rossendorf, Institute of Radiation Physics, Bautzner Landstraße 400, 01328 Dresden, Germany \\ ${ }^{3}$ Physikalisch-Technische Bundesanstalt, Department 6.4 - Ion and Neutron Radiation, Bundesallee 100, 38116 Braunschweig, \\ Germany
}

\begin{abstract}
A new experimental setup for the measurement of neutron scattering cross sections and angular distributions is currently being developed at the neutron time-of-flight facility GELINA, at the JRC-Geel. Up to 32 liquid organic scintillators are employed for the detection of neutrons scattered from a sample of the investigated material. The differential cross section is measured at eight different angles, and the angleintegrated cross section is obtained from the differential data by numerical integration. Two experiments for the study of scattering on iron were carried out, one at GELINA and the other at nELBE (HZDR). The first results for the angular distributions of elastic scattering in the neutron energy range from 2 to $6 \mathrm{MeV}$ are here presented and compared with evaluations from the major nuclear data libraries.
\end{abstract}

\section{Introduction}

Neutron scattering is the most important energy loss mechanism and the main moderation process for fast neutrons in nuclear reactors. Scattering cross sections significantly affect criticality and safety calculations, and double differential cross sections in particular are necessary for determining spatial distributions.

Iron is one of the main reactor structural materials: depending on the structure of the vessel, the volume fraction of iron can up to times higher than that of fuel. Being present in such large quantities, it heavily influences the neutron energy spectrum, the flux distribution and the reaction rates. Therefore, accurate scattering cross section are indispensable for reliable models. In the case of the Generation-IV Sodium-cooled Fast Reactors, for example, where the neutron leakage from the core is limited by means of stainless steel reflectors, the iron-56 scattering is one of the major sources of the overall uncertainty in the determination of the most important integral parameters (multiplication factor, reactivity coefficients, power distributions) [1]. The number of angular distribution experimental points in the current iron cross-section libraries for energies higher than $1 \mathrm{MeV}$ is quite limited. Furthermore, some discrepancies exist between evaluated and experimental integral crosssections [2]. For these reasons, iron-56 was included in the high priority list for nuclear data of the OECD-NEA [3] and it is one of the six nuclides for the pilot project of the CIELO (Collaborative International Evaluated Library Organization) collaboration [4].

A new experimental setup for the investigation of neutron scattering cross sections and angular distributions is currently being developed at the neutron time-of-flight facility GELINA. An array of up to 32 liquid organic scintillators is employed to detect the neutrons scattered from the investigated sample. The detector arrangement allows to measure the scattered neutron distributions at eight different angles and, simultaneously, to determine the total cross section. To date, the new setup was employed for two experiments for the study of scattering on natural iron in the fast neutron energy range, one at GELINA, at the European Commission's Directorate-General Joint Research Centre in Geel (JRC-Geel), and the other at nELBE, at the Helmholtz-Zentrum Dresden-Rossendorf (HZDR).

\section{Experimental setup}

GELINA (Geel Electron LINear Accelerator) is the pulsed white neutron source for high resolution neutron data measurements operated by the JRC-Geel [5]. The facility consists of four main elements: the electron linear accelerator, a compression magnet, a neutron producing target and the flight paths with the measurement stations. The linac produces electrons in pulses of typically $10 \mathrm{~ns}$ width (FWHM), at a repetition rate up to $800 \mathrm{~Hz}$, with energy varying linearly from $140 \mathrm{MeV}$ to $70 \mathrm{MeV}$. The pulses width is reduced by the compression magnet, a $360^{\circ}$ deflection dipole magnet that reduces the time spread inside the pulse to less than $1 \mathrm{~ns}$. The neutron source is a mercury cooled rotating target containing depleted uranium. When the electron beam strikes the target, neutrons with energies up to $20 \mathrm{MeV}$ are produced mainly via $(\gamma, \mathrm{n}),(\gamma, 2 \mathrm{n})$ and $(\gamma, \mathrm{F})$ reactions induced by the bremsstrahlung. In order to increase the number of neutrons with energy below $100 \mathrm{keV}$, two beryllium tanks filled with water are mounted above and below the target to act as moderators. GELINA serves 12 flight paths, and for each of them, two flux conditions are available: one optimised for energy below $100 \mathrm{keV}$ and the other for 
Table 1. Angles between the scintillators axis and the neutron beam direction, and corresponding weights for the application of the Gauss-Legendre quadrature rule (Eq. (1)). The uncertainty on the angles of $0.1^{\circ}$ is the accuracy achieved in the construction of the detector supporting frame.

\begin{tabular}{ccc}
\hline angle $\left(^{\circ}\right)$ & cosine & weight \\
\hline $163.8(1)$ & $-0.9603(5)$ & 0.1012 \\
$142.8(1)$ & $-0.7967(11)$ & 0.2224 \\
$121.7(1)$ & $-0.5255(15)$ & 0.3137 \\
$100.6(1)$ & $-0.1834(17)$ & 0.3627 \\
$79.4(1)$ & $0.1834(17)$ & 0.3627 \\
$58.3(1)$ & $0.5255(15)$ & 0.3137 \\
$37.2(1)$ & $0.7967(11)$ & 0.2224 \\
$16.2(1)$ & $0.9603(5)$ & 0.1012 \\
\hline
\end{tabular}

fast neutrons. These are obtained by placing shadow bars between the source and the given flight path to shield the neutrons coming either directly from the uranium target or from the moderators.

The setup here presented is installed along the flight path at $108^{\circ}$ from the electron beam, in the measurement cabin placed at the nominal distance of $30 \mathrm{~m}$ from the neutron source. It is the most backward flight path with respect of the electron beam direction, so it is where the self-attenuation of the uranium target is at its lowest and the neutron flux has the highest intensity. The energy range of interest is that of fast neutrons; therefore, the unmoderated neutron flux condition was selected.

The experimental setup consists of 32 liquid organic scintillators for the detection of the neutron scattered from a sample of the investigated material, and a ${ }^{235} \mathrm{U}$ fission chamber for the measurement of the incoming neutron fluence. The fission chamber contains eight $\mathrm{UF}_{4}$ deposits of $70 \mathrm{~mm}$ diameter, for a total ${ }^{235} \mathrm{U}$ areal density equal to $4.137(15) \mathrm{mg} / \mathrm{cm}^{2}$. The scintillators are cylindrical detectors; the liquid cells have $25.4 \mathrm{~mm}$ radius and $50.8 \mathrm{~mm}$ height. They are arranged in four sets of 8 detectors each: two sets of EJ301 of scintillators (NE213 equivalent, Scionix: model 51A51/2MQOE1-EJ301-NX) and two sets of EJ315 $\left(\mathrm{C}_{6} \mathrm{D}_{6}\right.$ detectors, Scionix: model 51A51/2MQOE1-EJ315-NX). Both kinds of scintillators are fast detectors, with a time resolution typically lower than $1 \mathrm{~ns}$. They allow neutron/photon separation via pulse shape analysis, so they are well suited for neutron time-offlight experiments. Both of them can be used for neutron spectrometry. Since at a given scattering angle the neutron energy after an elastic collision is different from the energy after an inelastic collision, the pulse height distribution of the scintillation detectors can be analysed to separate elastic scattering events from inelastic scattering.

Each detector of the four sets is placed at a specific angle with respect to the neutron beam direction; the exact value is reported in Table 1. These particular angles have been chosen in order to apply the Gauss-Legendre quadrature rule to determine the total cross section from the differential cross section. Their cosines correspond to the zeros of the Legendre polynomial of $8^{\text {th }}$ order; the cross section $\sigma$ can therefore be calculated by:

$$
\sigma=2 \pi \sum_{i=1}^{8} w_{i} \cdot \frac{d \sigma}{d \Omega}\left(\cos \theta_{i}\right)
$$

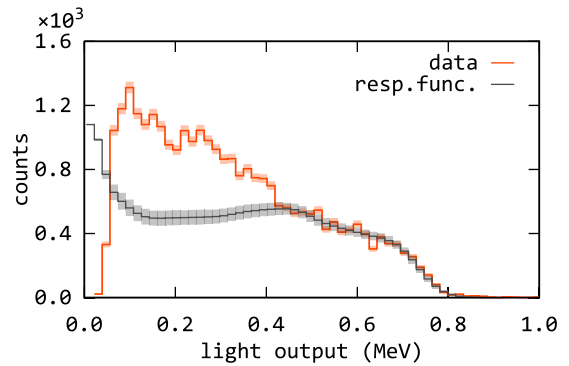

Figure 1. Experimental light output histogram measured with a EJ301 detector at $163.8^{\circ}$, compared to the simulated detector response. The histogram was obtained for neutrons with t.o.f. between $1213 \mathrm{~ns}$ and $1218 \mathrm{~ns}$, which corresponds to $2.641 \mathrm{MeV}-2.667 \mathrm{MeV}$ energy incident on the target and $2.460 \mathrm{MeV}-2.485 \mathrm{MeV}$ energy at the detector (elastically scattered neutrons). The detector response function was modelled for monoenergetic neutrons with $2.47 \mathrm{MeV}$ energy.

where $\frac{d \sigma}{d \Omega}\left(\cos \theta_{i}\right)$ is the differential cross section as function of the scattering angle $\theta_{i}$, and $w_{i}$ are the the weight factors reported in Table 1. The Gauss-Legendre quadrature rule is constructed in such a way that the sum in Eq. (1) leads the exact results of $\int_{-1}^{1} \frac{d \sigma}{d \Omega}(x) d x$ if $\frac{d \sigma}{d \Omega}(x)$ is a polynomial in $\mathrm{x}$ of $15^{\text {th }}$ order or less.

\section{Experiment on iron}

Two experiments were realized using as target for neutron scattering a $3 \mathrm{~mm}$ thick disk of natural iron, with an areal density of $0.205(4) \mathrm{g} / \mathrm{cm}^{2}$. The first experiment was carried out at nELBE, the neutron time-of-flight facility installed at the superconducting Electron Linac for beams with high Brilliance and low Emittance (ELBE) of the HZDR. At nELBE, neutrons are produced when the pulsed electron beam hits a liquid lead target, via bremsstrahlung induced $(\gamma, \mathrm{n})$ reactions. The collimator which defines the neutron beam is a steel tube with lead and polyethylene inserts, and it is installed at $95^{\circ}$ with respect to the primary electron beam. The experimental hall is separated from the neutron radiator by a $2.5 \mathrm{~m}$ thick wall of heavy and normal concrete. There, the detector setup is located at least at $3 \mathrm{~m}$ from the walls, ceiling and floor. For the experiment at nELBE, eight EJ301 detectors and eight EJ315 detectors were used, and the target was placed at 6.100(1) $\mathrm{m}$ from the neutron radiator. At GELINA, the full setup with 32 detectors was employed. In this case, the flight path length was of 27.037(3) $\mathrm{m}$.

In both experiments, the time-of-flight (t.o.f.) was measured by clocking the time between the accelerator reference signal, which is phase-locked to the electron bunches, and the signals from the detectors. The experimental t.o.f. is measured relative to the known arrival time of scattered bremsstrahlung photons. It is the sum of the t.o.f. of the incoming neutron travelling from the source to the scattering target, and the t.o.f. of the scattered neutron going from the target to the detector; it is a function of both the initial neutron energy and the energy that the neutron has after the scattering reaction. Due to the two body kinematics, the energy after a collision depends on the initial energy and on the scattering angle only: therefore, there is a one-to-one correspondence between 

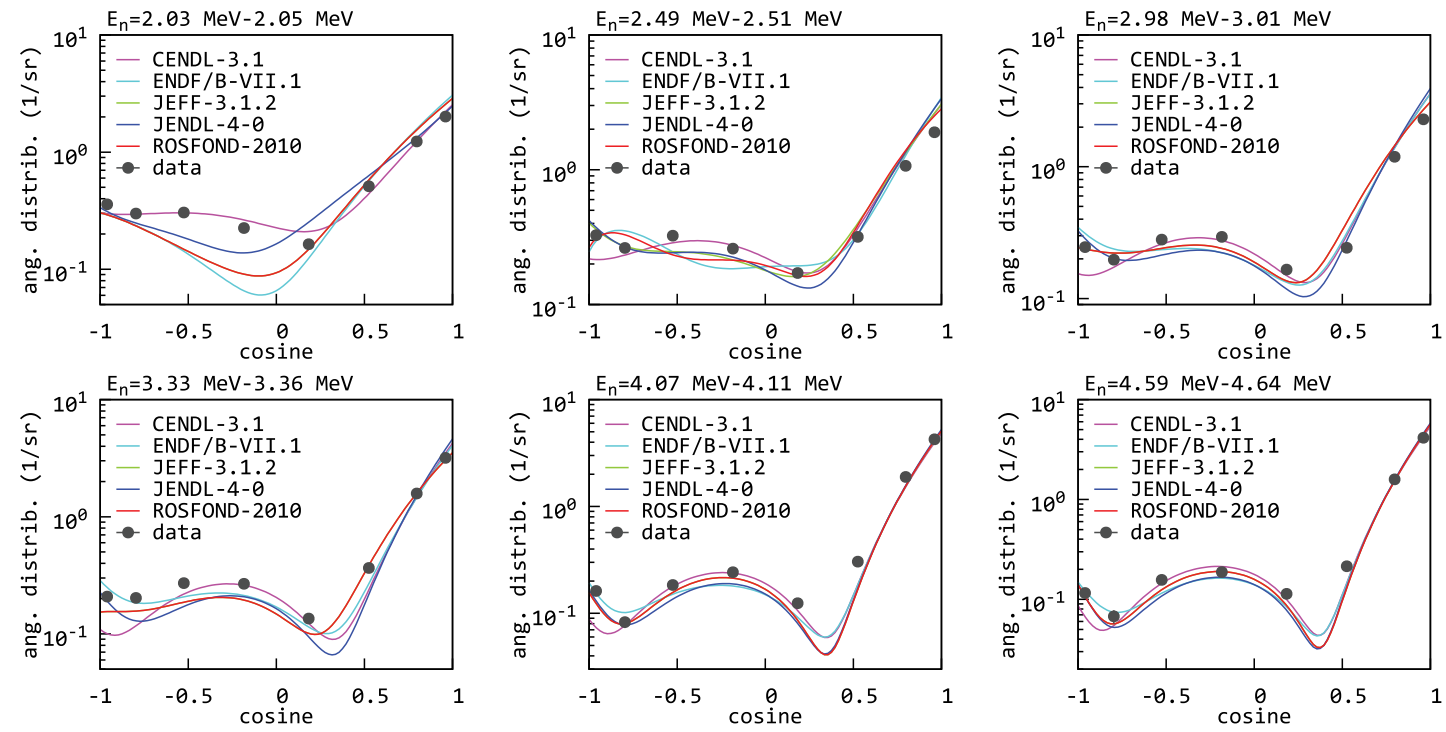

Figure 2. Angular distributions of neutron elastic scattering on iron as function of the cosine of the scattering angle in the laboratory frame of reference, for selected neutron incident energies ( $\mathrm{E}_{n}$ as indicated above each plot).

the t.o.f. measured with a fixed detector and neutron initial energy.

The first step in the data analysis is the separation of photon induced events from the neutron induced events via Pulse Shape Discrimination (PSD). For this, the well-established charge integration method [6] and the procedure described in [7] are employed. Two-dimensional histograms are then built by classifying the signals according to the t.o.f. and their total integrated charge. The linear scale of the charge is calibrated to obtain, as conventional, the light output expressed as equivalent electron energy (see [8] for the calibration procedure). The contribution of background neutrons (neutrons scattering in air or in the supporting frame) is determined and then removed by means of a "sample-out" measurement, i.e., a measurement run performed after removing the iron sample from the setup.

In Fig. 1, the light output spectrum, obtained by gating the two-dimensional histogram on the t.o.f. range from $1213 \mathrm{~ns}$ to $1218 \mathrm{~ns}$, is shown. These data were recorded at GELINA with a EJ301 detector in the most backward position $\left(163.8^{\circ}\right)$. If only elastic scattering is considered, the t.o.f. range corresponds to $2.641 \mathrm{MeV}$ $2.667 \mathrm{MeV}$ of neutron energy incident on the sample and to $2.460 \mathrm{MeV}-2.485 \mathrm{MeV}$ of neutron energy at the detector. The detection threshold of about $90 \mathrm{keV}$ (in electron energy) corresponds to a neutron energy of $0.7 \mathrm{MeV}$. The data are compared with the results of a simulation modelling the detector response to monoenergetic neutrons with $2.47 \mathrm{MeV}$ energy. The two distributions overlap only where the yield is given by just elastic scattering events. The low part of the spectrum (below $0.4 \mathrm{MeV}$ in Fig. 1) could be modelled by solving the relationship between t.o.f. and energy for inelastic scattering case, and by reproducing the detector response for the resulting values. For the sake of simplicity, however, only elastic scattering has been considered at first. The accuracy of the simulations was verified by comparing it with measurements of scattering from a graphite target. With carbon, below $4 \mathrm{MeV}$ of incident energy, only elastic scattering occurs, and the simulated spectra reproduce the measurements (in the limits of the uncertainties) over the whole light output range.

The detector response is obtained by modelling the scintillators using the particle transport code MCNP5 [9]. The energy deposited by the neutrons in the detectors sensitive volume is converted in light output using the parametric formula given in [10]. To take into account the finite resolution of the detectors, the results of the simulations are folded with a Gaussian resolution function, whose width is quadratic in the light output.

Fitting the detector response to monoenergetic neutrons, to the light-output experimental histogram (as in Fig. 1), yields the total number of collisions in which the secondary neutron is scattered in the direction of the detector for a given t.o.f. (or a given neutron incident energy). From this, and the iron sample areal density, the differential cross section is determined by computing the ratio to the incoming neutron fluence. The fluence is determined by analysing the fission fragment yield of the fission chamber according to the procedure described in [11].

The method for the separation of inelastic and elastic scattering is similar to that reported in [12]; the main difference lies in the way the detector response is determined. In [12], the response is obtained experimentally, with the advantage of not requiring any assumption on the detection efficiency. The model here presented needs to be experimentally validated; it has however the advantage of providing a way to extrapolate the number of events below the detection threshold.

\section{Results}

The data analysis procedure allows, in principle, the separation of both elastic and inelastic scattering. However, since the relation between t.o.f. and neutron incident energy is easier in case of elastic scattering, only this has been considered at first. In Fig. 2, the experimental angular distributions, obtained for selected energies between 2 and $5 \mathrm{MeV}$, are compared with the values reported by some of the major evaluated nuclear 


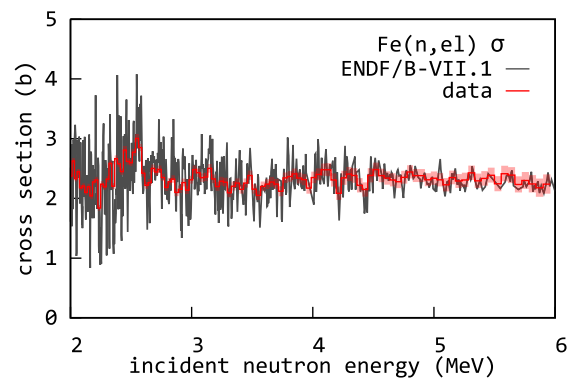

Figure 3. Cross section of neutron elastic scattering on iron.

data libraries. The total cross section, obtained for incident energies from 2 to $6 \mathrm{MeV}$, is shown in Fig. 3 .

As far as it regards the total cross section, there is overall good agreement between the experimental data and the ENDF/B-VII.1 library. The energy resolution of the data however does not allow to make any final statement on the details of the evaluation. For the same reason, it was not deemed necessary compare the data with more than one library.

As for the angular distributions, eight experimental points might not be sufficient to determine the whole Legendre expansion at all energies. However they are at least sufficient to extract the first order coefficient and partially solve the discrepancies between evaluations. Overall, none of the evaluations considered in Fig. 2 seems to perform better than the other in reproducing the data. The compatibility depends mostly on which energy is considered. In general, data and evaluations tend to disagree the most at backward angles and for energies below $3 \mathrm{MeV}$.

These are preliminary results from the analysis of only one set of EJ301 detectors. So, similarly as for total cross sections, also for the angular distributions it is difficult to qualify properly the performance of the single libraries compared with the experimental points. The comparison among different sets of detectors is an essential step in order to evaluate the accuracy of the results; and this is particularly relevant at backwards angles, where the low statistics due to the physics of the process imply unavoidably bigger uncertainties in the measurements.

\section{Conclusions}

A new experimental setup for the measurement of neutron scattering cross sections and angular distributions is currently being developed at the GELINA facility. Up to 32 liquid organic scintillators are employed to detected neutrons scattered from the target sample and to measure the differential cross section at eight different angles. The separation of elastic from inelastic scattering is achieved via neutron spectroscopy, and the total cross section is obtained via numerical integration.

Up to now, two experiments for the study of neutron scattering on iron were performed at different t.o.f. facilities, GELINA and nELBE. The preliminary results for the elastic scattering angular distribution were compared with the major nuclear data libraries in the incident neutron energy range from $2 \mathrm{MeV}$ to $6 \mathrm{MeV}$. The biggest discrepancies among evaluation and preliminary data are mostly found for backward scattering angles and for energies below $3 \mathrm{MeV}$. The contribution of elastic scattering is the easiest to isolate and, at first, only that has been considered. In principle, the same technique can be adapted to investigate also inelastic scattering.

\section{References}

[1] M. Salvatores, R. Jacqmin, WPEC-26 final report, OECD NEA (2008)

[2] M. Wenner, A. Haghighat, J. Adams, A. Carlson, S. Grimes, T. Massey, Nucl. Sci. Eng. 170, 207 (2012)

[3] OECD NEA, Nuclear Data High-Priority Request List, http://www . oecd-nea.org/dbdata/hprl/

[4] M. Chadwick, E. Dupont, E. Bauge, A. Blokhin, O. Bouland, D. Brown, R. Capote, A. Carlson, Y. Danon, C.D.S. Jean et al., Nucl. Data Sheets 118, 1 (2014)

[5] W. Mondelaers, P. Schillebeeckx, Notiziario neutroni e luce di sincrotrone 11(2), 19 (2006)

[6] F. Brooks, Nucl. Instrum. Methods 4, 151 (1959)

[7] J. Polack, M. Flaska, A. Enqvist, C. Sosa, C. Lawrence, S. Pozzi, Nucl. Instrum. Methods Phys. Res. A 795, 253 (2015)

[8] H. Klein, S. Neumann, Nucl. Instrum. Methods Phys. Res. A 476, 132 (2002)

[9] X-5 Monte Carlo Team, MCNP - A General Monte Carlo N-Particle Transport Code, Version 5, LANL (2003), 1A-UR-03-1987

[10] N. Kornilov, I. Fabry, S. Oberstedt, F.J. Hambsch, Nucl. Instrum. Methods Phys. Res. A 599, 226 (2009)

[11] C. Rouki, P. Archier, C. Borcea, C. De Saint Jean, J. Drohé, S. Kopecky, A. Moens, N. Nankov, A. Negret, G. Noguère et al., Nucl. Instrum. Methods Phys. Res. A 672, 82 (2012)

[12] A. Daskalakis, E. Blain, B. McDermott, Y. Danon, in AccApp '15 (Washington, DC, 2015), pp. 374-379 